\title{
Microscopic velocity field measurements inside a regular porous medium adjacent to a low Reynolds number channel flow
}

Citation for published version (APA):

Terzis, A., Zarikos, I., Weishaupt, K., Yang, G., Chu, X., Helmig, R., \& Weigand, B. (2019). Microscopic velocity field measurements inside a regular porous medium adjacent to a low Reynolds number channel flow. Physics of Fluids, 31(4), [042001]. https://doi.org/10.1063/1.5092169

DOI:

10.1063/1.5092169

Document status and date:

Published: 01/04/2019

Document Version:

Publisher's PDF, also known as Version of Record (includes final page, issue and volume numbers)

Please check the document version of this publication:

- A submitted manuscript is the version of the article upon submission and before peer-review. There can be important differences between the submitted version and the official published version of record. People interested in the research are advised to contact the author for the final version of the publication, or visit the $\mathrm{DOI}$ to the publisher's website.

- The final author version and the galley proof are versions of the publication after peer review.

- The final published version features the final layout of the paper including the volume, issue and page numbers.

Link to publication

\footnotetext{
General rights

- You may freely distribute the URL identifying the publication in the public portal. follow below link for the End User Agreement:

www.tue.nl/taverne

Take down policy

If you believe that this document breaches copyright please contact us at:

openaccess@tue.nl

providing details and we will investigate your claim.
}

Copyright and moral rights for the publications made accessible in the public portal are retained by the authors and/or other copyright owners and it is a condition of accessing publications that users recognise and abide by the legal requirements associated with these rights.

- Users may download and print one copy of any publication from the public portal for the purpose of private study or research.

- You may not further distribute the material or use it for any profit-making activity or commercial gain

If the publication is distributed under the terms of Article 25fa of the Dutch Copyright Act, indicated by the "Taverne" license above, please 


\section{Microscopic velocity field measurements inside a regular porous medium adjacent to a low Reynolds number channel flow}

Cite as: Phys. Fluids 31, 042001 (2019); https://doi.org/10.1063/1.5092169

Submitted: 08 February 2019 . Accepted: 14 March 2019 . Published Online: 02 April 2019

A. Terzis, I. Zarikos, K. Weishaupt, G. Yang (D), X. Chu (D), R. Helmig, and B. Weigand
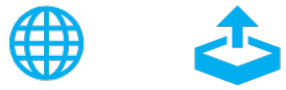

View Online

\section{ARTICLES YOU MAY BE INTERESTED IN}

Aerodynamic forces and vortical structures of a flapping wing at very low Reynolds numbers

Physics of Fluids 31, 041901 (2019); https://doi.org/10.1063/1.5089804

Steady streaming viscometry of Newtonian liquids in microfluidic devices

Physics of Fluids 31, 041701 (2019); https://doi.org/10.1063/1.5092634

Experimental study of single-particle trapping mechanisms into microcavities using microfluidics

Physics of Fluids 31, 042002 (2019); https://doi.org/10.1063/1.5081918

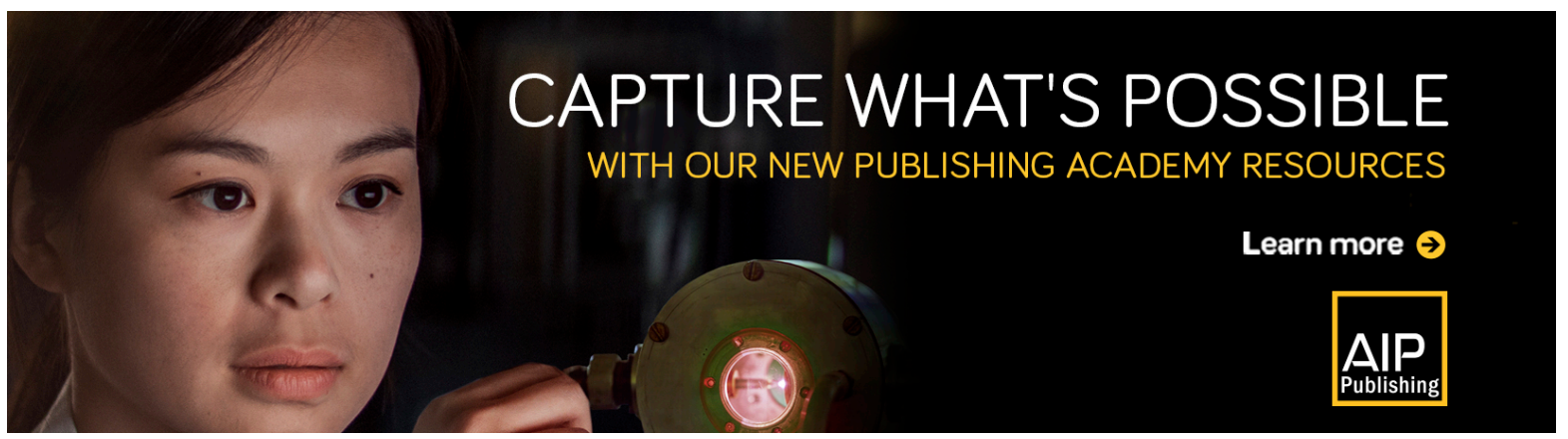




\title{
Microscopic velocity field measurements inside a regular porous medium adjacent to a low Reynolds number channel flow
}

\author{
Cite as: Phys. Fluids 31, 042001 (2019); doi: 10.1063/1.5092169 \\ Submitted: 8 February 2019 - Accepted: 14 March 2019 • \\ Published Online: 2 April 2019
}

A. Terzis, ${ }^{1, a)}$ I. Zarikos, ${ }^{2}$ K. Weishaupt, ${ }^{3}$ G. Yang, ${ }^{4}$ (D) X. Chu, ${ }^{1}$ (D) R. Helmig, ${ }^{3}$ and B. Weigand

\begin{abstract}
AFFILIATIONS
${ }^{1}$ Institute of Aerospace Thermodynamics (ITLR), University of Stuttgart, 70569 Stuttgart, Germany

${ }^{2}$ Multiscale Engineering Fluid Dynamics (MEFD), Eindhoven University of Technology, 5600 MB Eindhoven, The Netherlands

${ }^{3}$ Department of Hydromechanics and Modelling of Hydrosystems (IWS), University of Stuttgart, 70569 Stuttgart, Germany

${ }^{4}$ Institute of Refrigeration and Cryogenics, Shanghai Jiao Tong University (SJTU), 200240 Shanghai, China
\end{abstract}

a) Author to whom correspondence should be addressed: alexandros.terzis@me.com

\begin{abstract}
This study examines experimentally the hydrodynamic interaction between a regular porous medium and an adjacent free-flow channel at low Reynolds numbers $(R e<1)$. The porous medium consists of evenly spaced micro-structured rectangular pillars arranged in a uniform pattern, while the free-flow channel features a rectangular cross-sectional area. The overall arrangement comprises a polydimethylsiloxane microfluidic model where distilled water, doped with fluorescent particles, is the examined fluid. Using micro-particle image velocimetry, single-phase quantitative velocity measurements are carried out at the pore scale to reveal the microscopic characteristics of the flow for such a coupled system. Interfacial velocity-slip and stress-jump coefficients are also evaluated with a volume-averaging method based on the Beavers-Joseph and Ochoa-Tapia-Whitaker models, respectively. The results show that, from a microscopic point of view, parallel flow at the interface is not obtained due to the periodically generated U-shaped flow profile between the interface pillars. However, the interface coefficients show no sensitivity to moderate flow angles. The highly resolved experimental information obtained in this study can also be used for the validation of numerical models providing a unique dataset for free-flow and porous media coupled systems.
\end{abstract}

Published under license by AIP Publishing. https://doi.org/10.1063/1.5092169

\section{INTRODUCTION}

The interaction between a permeable solid and an adjacent free-flow is of central interest in many natural and industrial processes and on multiple scales. Particular examples include atmospheric flows that govern evaporation rates from soil matrices, exchange processes of therapeutic agents in biological tissues, ${ }^{2}$ water management in fuel cells, ${ }^{3}$ transpiration cooling, ${ }^{4}$ drying processes in food industry, ${ }^{5}$ or even noise reduction at the trailing edge of aircraft wings. ${ }^{6}$ A detailed knowledge of the underlying flow physics is therefore crucial for the proper description of the flow domain, as well as the optimisation of the associated technological applications.

From a macroscopic point of view, the fluid motion through an isotropic and homogeneous porous medium is governed by Darcy's law (or Brinkmann's equation ${ }^{8}$ ), while the Navier-Stokes equations, being microscopic, are typically applied to describe the free-flow regime, often in their simplified Stokes' approximation due to the small length-scales, and hence, low Reynolds numbers. However, this leads to mathematical difficulties arising from the coupled system of equations of different orders in different regions. The use of Brinkman's extension over the entire flow domain ("single-domain" approach) may simplify the situation through a transition zone with spatial variation of effective properties, e.g., permeability, viscosity, and porosity. Nevertheless, the determination of such parameters is intricately obtained, ${ }^{9,10}$ while the applicability of single-domain methods at high-porosity media is still under consideration. ${ }^{11-14}$ Furthermore, two-phase flow problems require that the free-flow and the porous medium are separately treated constituting the so-called "two-domain" modeling approaches. ${ }^{15}$ Here, the quantification of mass, momentum, and energy transport fluxes is a challenging task that depends on the use of an appropriate 
hydrodynamic boundary condition at the interface between the two distinct regions. ${ }^{16}$

The foremost boundary condition, initially proposed by Beavers and Joseph, ${ }^{18}$ introduces a dimensionless slip coefficient to relate the interface velocity gradient to a tangential slip velocity as follows:

$$
\left.\frac{\partial\langle u\rangle}{\partial y}\right|_{y=0^{-}}=\frac{\alpha_{\mathrm{BJ}}}{\sqrt{k}}\left(\left.\langle u\rangle\right|_{y=0}-U_{\mathrm{D}}\right),
$$

where $\left\langle\left. u\right|_{y=0}\right.$ and $U_{\mathrm{D}}$ denote the volumetric-averaged interfacial and Darcy velocities, respectively; $k$ is the permeability of the porous material, and $\alpha_{\mathrm{BJ}}$ is the Beavers and Joseph slip coefficient. Saffman ${ }^{19}$ proposed to neglect $U_{D}$ due to the relatively small magnitude compared to the interface boundary. This condition, which is schematically shown in Fig. 1, has been extensively studied in the literature demonstrating the high sensitivity of $a_{\mathrm{BJ}}$ to various parameters, such as the interface location, ${ }^{19-22}$ the fluid properties, ${ }^{23,24}$ the flow conditions, ${ }^{21,25}$ the free-flow confinement, ${ }^{26,27}$ and the surface microstructure. ${ }^{21,24}$ An alternative approach, proposed by OchoaTapia and Whitaker, ${ }^{28,29}$ allows the transition from the microscopic to the mesoscopic description of the problem through a volumeaveraging method, and then to the macroscopic one through the introduction of a "stress-jump" boundary condition. The interface momentum transfer is then given by the following expression:

$$
\left.\frac{1}{\phi} \frac{\partial\langle u\rangle}{\partial y}\right|_{y=0^{+}}-\left.\frac{\partial\langle u\rangle}{\partial y}\right|_{y=0^{-}}=\left.\frac{\beta}{\sqrt{k}}\langle u\rangle\right|_{y=0}
$$

where $\phi$ is the porosity of the porous material and $\beta$ is a dimensionless constant. Similar to $\alpha_{\mathrm{BJ}}$, the a priori determination of $\beta$ is very complex with typical values varying in the order of one, and obtaining both positive and negative signs which are determined by the surface of a given material. ${ }^{30,31}$ Apparently, the two-domain interface boundary conditions involve coefficients $\left(\alpha_{\mathrm{BJ}}, \beta\right)$ whose physical interpretation is not adequately understood, and their appropriate use requires separate readjustments for each individual flow problem. For a more detailed overview, the reader may also refer to literature sources that extend the aforementioned classical concepts to multiphase flow conditions. ${ }^{32-35}$

Macroscopic numerical models are, in general, inadequate to account for the detailed pore scale flow fields that can also be

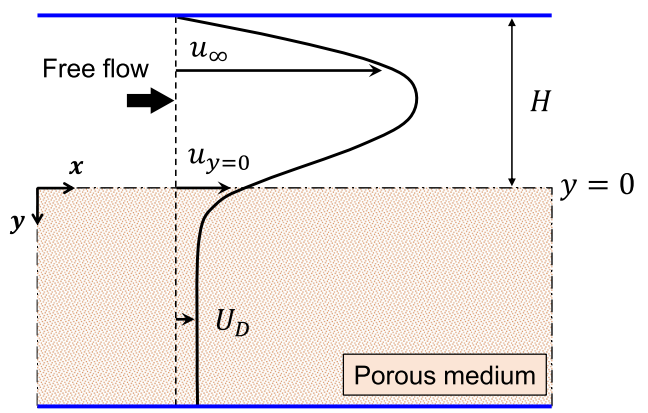

FIG. 1. Velocity profile for a flow over a porous medium. $U_{D}$ is the Darcy velocity inside the porous medium and far from the interface, and $\left.u\right|_{y=0}$ is the slip velocity between the two distinct regions. Inspired by Wu and Mirbod. ${ }^{1}$ characterised by viscous eddies and flow separation at sharp corners, even at creeping flow conditions. ${ }^{36-38}$ The knowledge of the microscopic flow field in a porous medium and free-flow coupled system is however the key for gaining insight into the developing models, as well as the pore scale physical processes, such as convective transport. For example, Direct Numerical Simulations (DNS) showed that different topological features exist in a porous medium, ${ }^{39}$ while their further influence on convective transport from the porous surfaces has been only numerically evaluated. ${ }^{40,41}$ Quantitative measurements of the microscopic velocity field in the vicinity of the interface are, however, very difficult to obtain. The majority of experimental studies have focused on high-porosity media with void spaces of more than $90 \%$, e.g., $0.93,{ }^{42} 0.9-0.975,{ }^{43}$ and $0.95-0.99,{ }^{17}$ while the porous material was adjacent to a nearly open channel flow. Using laser Doppler anemometry (LDA), ${ }^{42}$ pressure probes, ${ }^{44}$ and particle image velocimetry (PIV) techniques, ${ }^{10,43,45-47}$ the objective was to reveal the velocity profiles near the interface, evidencing the existence of a slip velocity at different flow conditions. However, in all the above-mentioned studies, the hydraulic diameters of the pores were well inside the meso-/macroscopic scale, e.g., 5$10 \mathrm{~mm},{ }^{10} 3.18 \mathrm{~mm},{ }^{43,45} 2-6 \mathrm{~mm},{ }^{46}$ and $1.59-4.56 \mathrm{~mm},{ }^{47}$ being at a certain significant distance from the microscopic world. Evidently, there is limited experimental information regarding the microscopic flow field that will provide knowledge towards the generalisation and the physical meaning of the aforementioned interface boundary conditions, including their semi-empirical velocity slip and stress jump coefficients.

The objective of this paper is therefore twofold: to reveal the microscopic velocity field of an entire porous medium that is adjacent to a channel flow and to derive the interface coefficients for the Beavers-Joseph-Saffman and Ochoa-Tapia-Whitaker boundary conditions. To achieve this, single-phase microfluidic experiments are carried out at low Reynolds numbers in a model of evenly spaced micro-structured rectangular pillars that constitute a regular porous structure of $75 \%$ porosity. The flow through the porous medium originates from the adjacent free-flow channel. This geometry is practical since it can be easily represented by computational tools. Hence, apart from the great fundamental interest, the results of this study can be further used for the validation of numerical models. The paper is organised as follows: Sec. II presents the experimental setup, the instrumentation, and the methodology, while Sec. III is classified into three parts. The description of the macro- and microscopic flow fields is given in Secs. III A and III B, and the derivation of the associated coefficients for the interface boundary conditions is given in Sec. III C.

\section{MATERIALS AND METHODS}

\section{A. Microfluidic model and experimental procedure}

Figure 2 shows a schematic of the microfluidic model, which was made of polydimethylsiloxane (PDMS) and fabricated via softlithographic techniques. ${ }^{4-51}$ The model comprises a channel flow with a rectangular cross-sectional area of $2000 \times 200 \mu \mathrm{m}^{2}$, which is adjacent to a porous structure that consists of $80 \times 20$ evenly spaced and finite-depth square pillars of $240 \mu \mathrm{m}$ size. The porosity of the porous structure is therefore 0.75 , while the thickness of the model equals the channel depth, e.g., $200 \mu \mathrm{m}$. PDMS micromodels have 


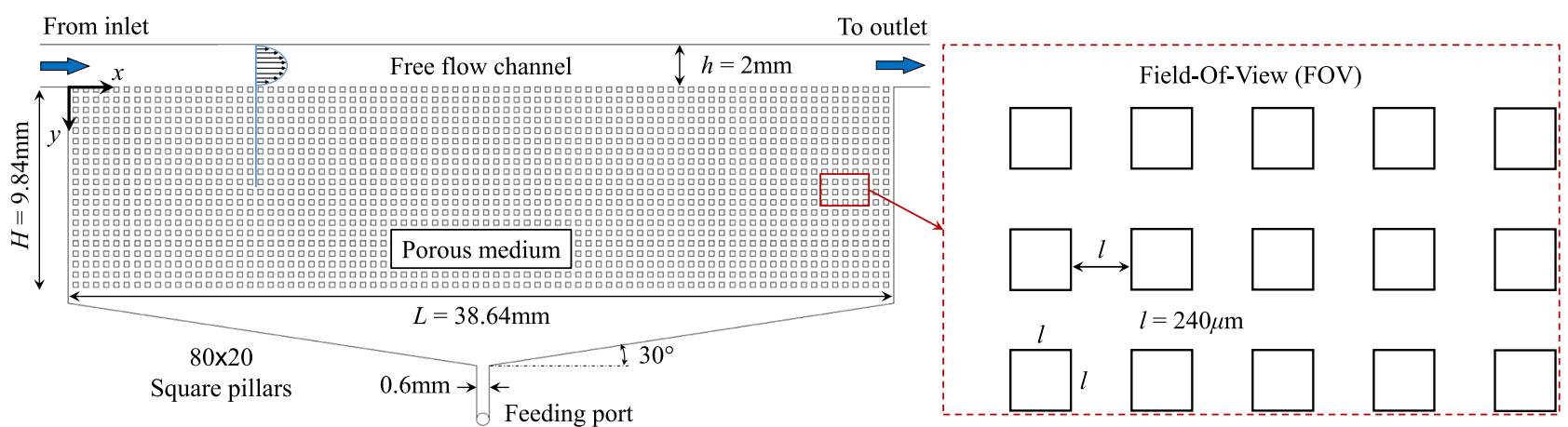

FIG. 2. (Left) Schematic illustration of the polydimethylsiloxane (PDMS) microfluidic model that consists of a rectangular channel flow that is adjacent to $80 \times 20$ evenly spaced square pillars. (Right) Typical field-of-view during the micro-PIV experiments.

also been used to study electro-kinetics ${ }^{52-54}$ and dispersion effects in porous media. ${ }^{55,56}$

The porous medium was initially saturated by dispensing through the feeding port a $50 \%$ (by volume) water-ethanol binary mixture facilitating the saturation process. This was due to the reduced surface tension that resulted in significant wetting on the low-energy PDMS surfaces. As soon as the model was completely saturated, the feeding port was blocked and distilled water $(72.8 \mathrm{mN} / \mathrm{m}, 1 \mathrm{mPa} \mathrm{s})$ was delivered for $30 \mathrm{~min}$ from the inlet of the model eliminating the percentage composition of ethanol. Then, doped water was inserted through a 3-way stopcock valve, introducing the fluorescent particles in the flow. Once the particles were diffused over the complete micromodel, the free-flow velocity was regulated by a Harvard Apparatus syringe pump (Model Pump 11 Elite Series) to meet the requirements of the desirable volumetric flow. The Reynolds numbers, based on the pillar size $(l)$ and channel free-stream velocity $\left(\bar{u}_{o}\right)$, were varied from 0.14 to 0.33 .

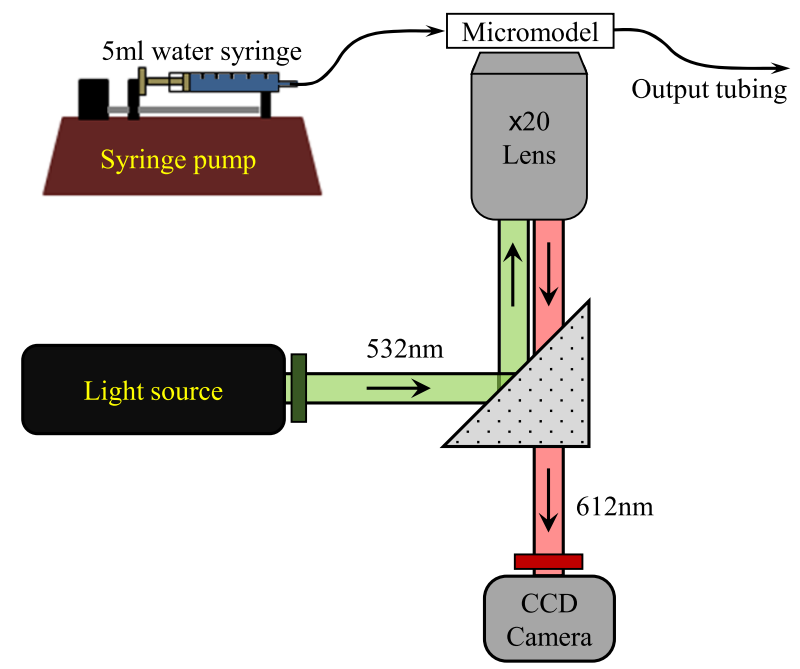

FIG. 3. Micro-PIV arrangement indicating the imaging process and the flow control system.

\section{B. Velocity measurements}

The pore scale velocity distributions were obtained with microParticle Image Velocimetry ( $\mu$ PIV $)^{57,58}$ using a LaVision micro-PIV system that is embedded in a Carl Zeiss Axio Observer Z1. The distilled water was doped with fluorescent Rhodamine $6 \mathrm{G}$ particles of $2 \mu \mathrm{m}$ diameter and $1005 \mathrm{~kg} / \mathrm{m}^{3}$ density. The particles were excited at a wavelength of $542 \mathrm{~nm}$ through a Carl Zeiss HBO 100 illuminator and identified at their emitting wavelength $(612 \mathrm{~nm})$ by an IDS CMOS camera (UI-3180CP, $2592 \times 2048$ pixels), as shown in Fig. 3 . Each Field-Of-View (FOV) consists of five and three pillars in $x$ and $y$-directions, respectively, as highlighted in Fig. 2. The complete coverage of the model was therefore achieved by $16 \times 8$ experiments, comprising 116 individual velocity scans. The velocity vectors were determined from consecutive image pairs of the video sequences, while the frame rate was adjusted depending on the flow conditions so that the maximum particle displacement never exceeded one-quarter of the final interrogation window, eliminating peaklocking effects. ${ }^{59,60}$ The velocity correlations were obtained by reducing $75 \%$ of the initial interrogation areas with a triple-pass FFT window deformation algorithm in MATLAB. ${ }^{61}$ An initial window size of $128 \times 128$ pixels was reduced to $32 \times 32$ pixels providing velocity vectors with a spatial resolution of approximately $20 \times 20 \mu \mathrm{m}^{2}$. The length of each individual pore space is therefore represented by approximately 12 velocity vectors. An uncertainty quantification based on cross-correlation statistics and the differences between the two consecutive images to be matched ${ }^{62}$ indicated that the particle displacement field is always estimated with an error below $6 \%$. More details about the velocity measurements can be found elsewhere.

\section{RESULTS AND DISCUSSION}

\section{A. Macroscopic flow field}

\section{Contour plots}

Figure 4 shows the $v$-velocity component and the flow angle contour plots over the entire porous medium and the free-flow region at a $R e_{l}=0.14$. Since the advective inertial forces are smaller compared to viscous forces, the flow enters the porous structure to bypass the free-flow channel at $x / l=0$ and escapes at the 


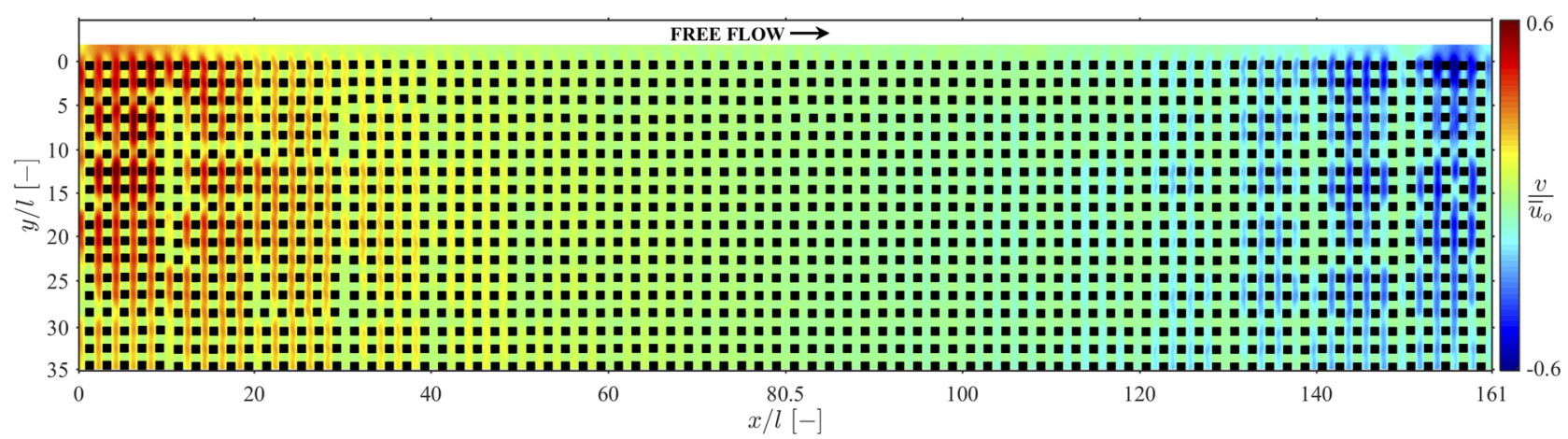

(a)

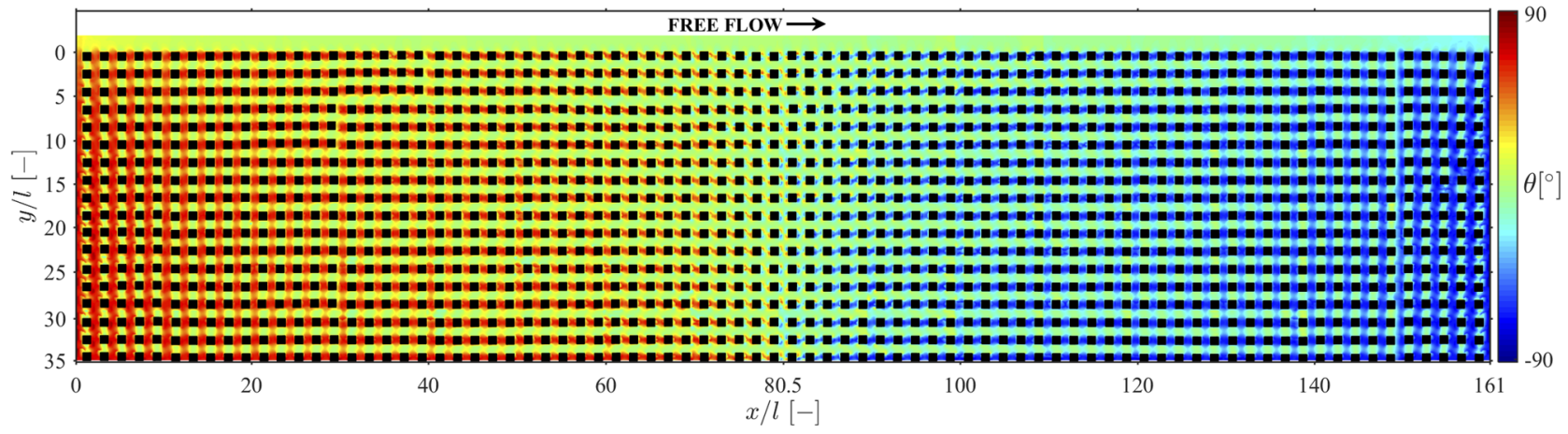

(b)

FIG. 4. (a) $v$-velocity component (y-direction) $\left(v / \bar{u}_{o}\right)$ and (b) flow angle $\left[\theta\left(^{\circ}\right)\right]$ contour plots over the complete micromodel area. $R e_{l}=0.14$.

downstream endwall $(x / l=161)$ in analogy to low Reynolds number flows over cavities. ${ }^{64,65}$ In particular, the maximum level of inward and outward porous medium velocity $(v)$ is about $60 \%$ of the average streamwise channel velocity $\left(\bar{u}_{o}\right)$ indicating the intensity of the vertical flow motion in these regions. In the streamwise direction, the $v$-velocity magnitude is gradually reduced until reaching zero values in the middle of the model $(x / l=80.5)$, as shown in Fig. 4(a). Therefore, the flow in the porous structure within the first 10 pillar rows from the interface $(y / l=0)$ is macroscopically parallel to the free-flow between $x / l=60$ and 100. However, at larger depths, e.g., $y / l>20$, a V-shaped flow distribution can be observed, especially in the flow angle contour of Fig. 4(b). This is attributed to the triangular reservoir at the bottom of the micromodel (see Fig. 2) where the flow experiences lower pressure drop, and hence, an easier flow path influences the surrounding fluid motion. Nevertheless, the overall macroscopic flow pattern demonstrates a typical U-shaped Stokes distribution where in the middle of the micromodel parallel flow is satisfactorily obtained.

\section{Local velocity distributions}

Figure 5 shows the local streamwise distributions of the velocity components for two different vertical locations: at the interface $(y / l=0)$ and the throat of the first pillar row $(y / l=0.5)$. The velocity component distributions can be generally classified into three regions: (I) the flow enters the porous structure from $x / l=0$ to 60 ,
(II) the porous media flow is parallel to the free-flow from $x / l=60$ to 100 , and (III) the flow escapes from the porous structure towards the channel flow from $x / l=100$ to 161 .

In Fig. 5(a), the $v$-velocity magnitude shows initially intensive fluctuations according to the position of the pillars at $y / l=0$, while the positive sign of $v$ shows that part of the main flow enters the porous structure. As the flow develops, the magnitude of $v$ is gradually reduced until the middle of the model at $x / l=80.5$, and the corresponding fluctuations are significantly smaller. In particular, their amplitude is less than $0.04 \mathrm{~mm} / \mathrm{s}$ between $x / l=60$ and 100 with both positive and negative values, signifying a more complex flow situation that will be examined in Sec. III B. This is the region where the flow in the porous structure is macroscopically parallel to the channel flow, and hence, dominated by the $u$-velocity component. At further downstream positions, the $v$-velocity magnitude is progressively increased in the opposite direction since the flow escapes from the porous material and the corresponding fluctuations are enhanced. Directly below the interface, at $y / l=0.5 l$, similar trends are observed. However, the difference between the inward/outward velocity regions and the middle of the micromodel is more pronounced. More specifically, the $v$-velocity magnitude is larger at the edges of the micromodel, indicating that the flow is accelerated as passing through the pillars, and nearly zero between $x / l=60$ and 100 , demonstrating a low momentum region.

The streamwise $u$-velocity component, shown in Fig. 5(b), indicates strong similarities with the spanwise one. The distribution of 


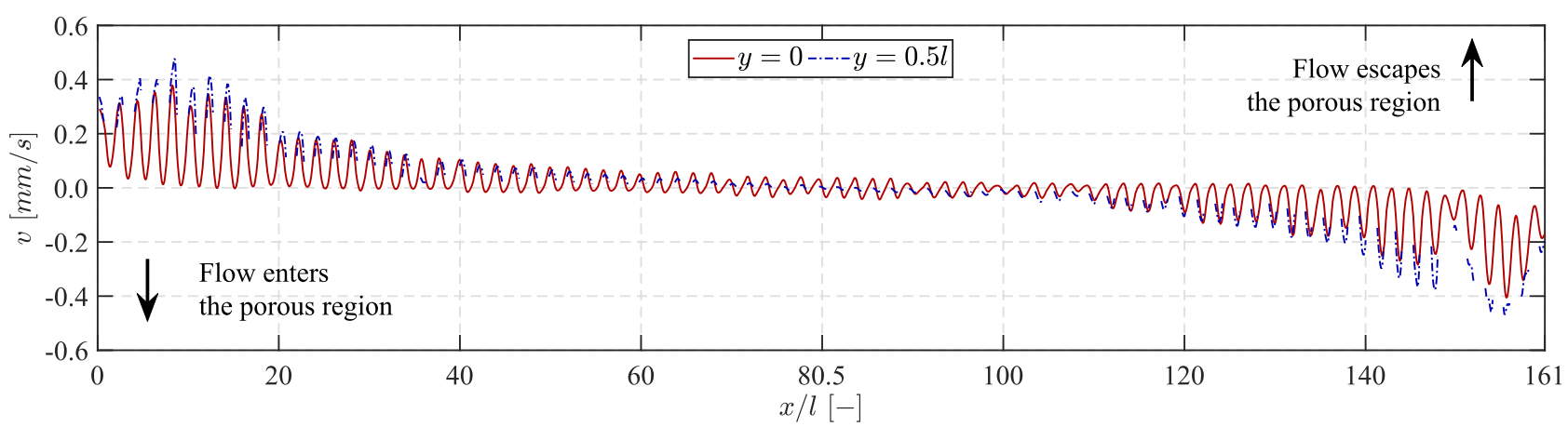

(a)

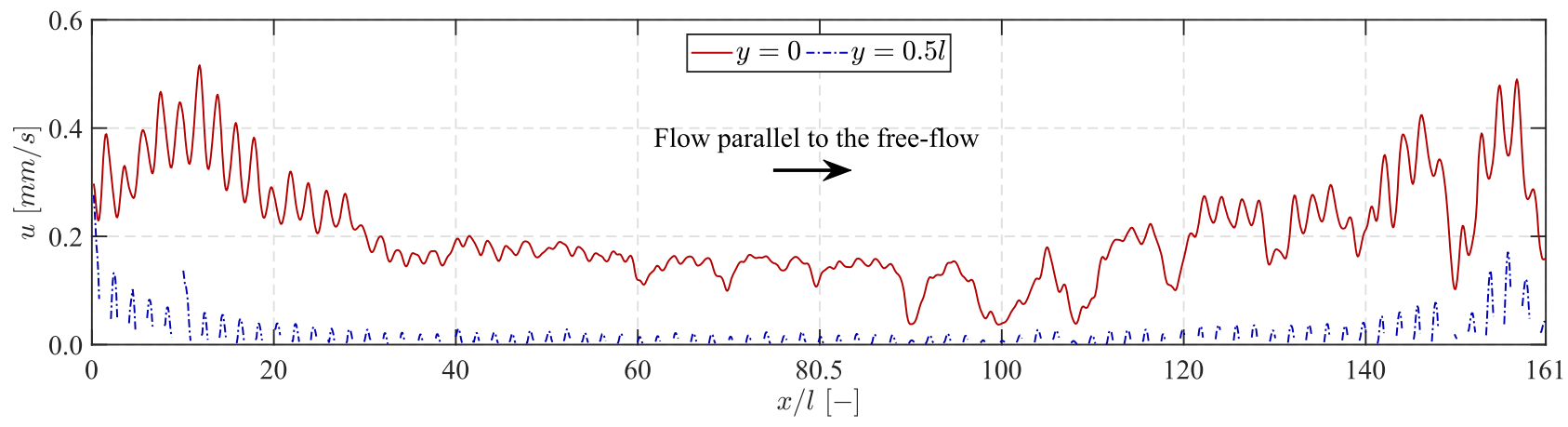

(b)

FIG. 5. Local velocity component distributions at the interface region $(y=0)$ between the free-flow and the porous structure. $u$ and $v$ are the velocity components in $x$ - and $y$-directions, respectively. (a) $v$-velocity component and (b) $u$-velocity component.

$u$ at $y / l=0$ is characterised by large fluctuations at the inlet and the outlet of the micromodel, while its magnitude is gradually reduced to fluctuate around $0.17 \mathrm{~mm} / \mathrm{s}$ in the region of parallel flow. At $y / l=0.5$, a similar distribution is observed; however, the $u$-velocity magnitude is significantly smaller compared to the interface. In particular, in the middle of the micromodel $(x / l=60-100), u$ is $90 \%$ lower compared to $y / l=0$ with a fluctuation amplitude less than $0.01 \mathrm{~mm} / \mathrm{s}$. Since the $v$-velocity in this region is also very small, this indicates a very low momentum region in the middle of the model, and between the pillars. Due to the similarity between the distribution of the two velocity components, one can say that the flow is generally decelerated until the middle of the micromodel and then it is accelerated again towards the outlet. This is a consequence of the constant massflow ratio in the streamwise direction and the triangular bottom of the model that increases locally the global cross-sectional area of the model.

\section{B. Microscopic flow field}

Figure 6 highlights the areas of the porous medium that are microscopically examined in Figs. 7 and 8. These include the $v$-velocity field and the corresponding velocity vectors in the vicinity of the interface $(y=0)$ for three different streamwise locations, that are $\mathrm{A}, \mathrm{B}$, and $\mathrm{C}$, as well as region $\mathrm{D}$ which is inside the porous medium.
In region $\mathrm{A}$, which is close to the inlet, Fig. 7(a) shows an oblique flow at the interface as the channel flow enters the porous medium. However, the direction of the flow is perpendicular to the free-flow channel at the void spaces between the pillars in the $x$-direction. In addition, the vertical velocity is clearly decelerated from pillars 12 to 19 , while similar trends are observed for the 2nd pillar row. The maximum vertical velocity component is about $45 \%$ of the streamwise channel velocity in this region indicating the significant momentum transport in the vertical direction.

In the middle of the micromodel (region B), the flow is macroscopically parallel to the channel flow. However, Fig. 7(b) indicates

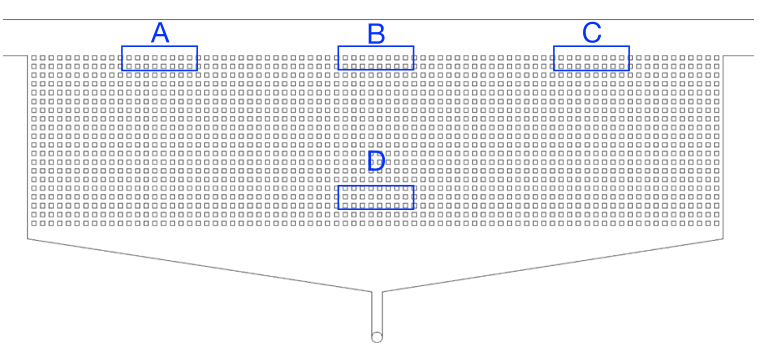

FIG. 6. Porous medium regions examined in Figs. 7 and 8. 


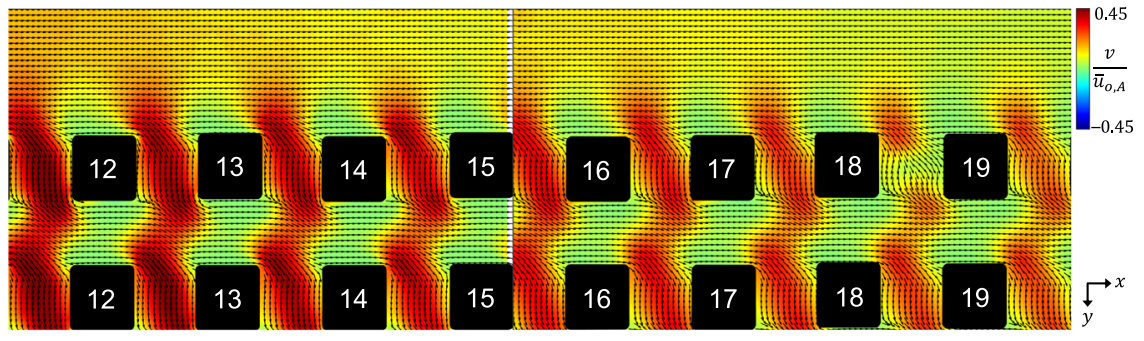

(a)

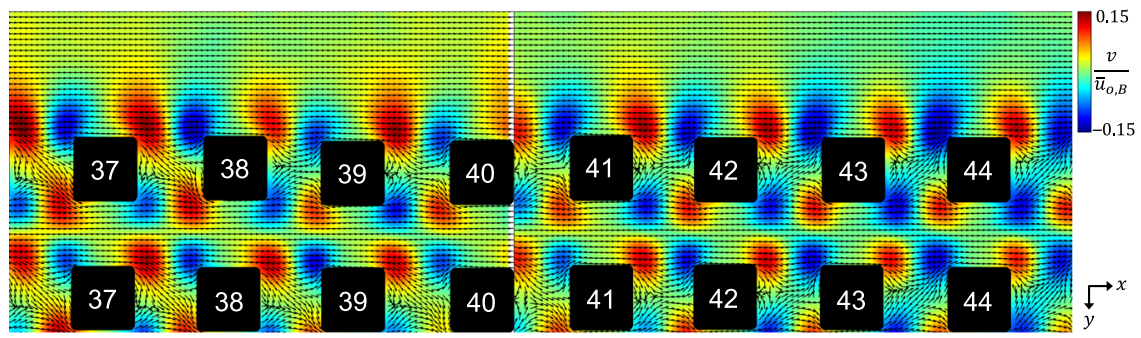

(b)

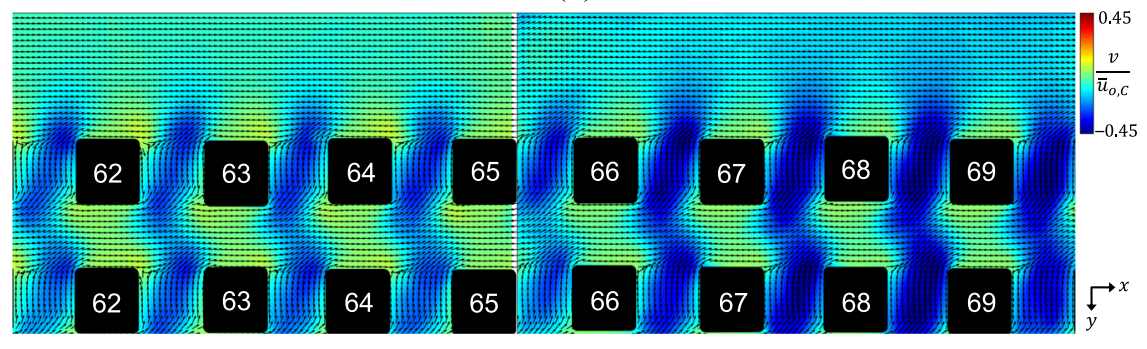

(c)

that parallel flow at the interface does not exist. Instead, the flow goes in and out of the porous medium by continuously crossing the interface location. Between the interface pillars, and due to the low Reynolds numbers, the flow generates typical Stokes U-shaped flow profiles. As a result, between the pillars in the $x$-direction, especially between pillars 38 and 42 , the velocity vectors indicate stagnant flow conditions at $y=0.5 l$. The dynamic behavior of the flow is similar for both pillar rows, although the overall intensity of the velocity fluctuations is less pronounced for the second row. Overall, the maximum $v$-velocity magnitude is about $15 \%$ of the streamwise channel flow in this region indicating significantly less momentum transport between the two regions compared to the inlet of the micromodel.
In region $\mathrm{C}$, which is close to the outlet of the porous medium, Fig. 7(c) shows the opposite trend compared to Fig. 7(a). The flow escapes the porous structure with an upward motion and an oblique direction compared to the channel flow. The $v$-velocity component is therefore accelerated in the streamwise direction with the maximum values reaching $45 \%$ of the freestream channel velocity. Therefore, one can say that region $\mathrm{C}$ is actually a flip mirror of region $\mathrm{A}$ with the middle of the micromodel being the symmetry plane.

Regarding the velocity field inside the porous medium (region D), Fig. 8 shows that the flow distribution is similar to the interface location, especially in the region between the pillars 39 and 41 . The flow goes in and out of the pore spaces with a $v$-velocity magnitude

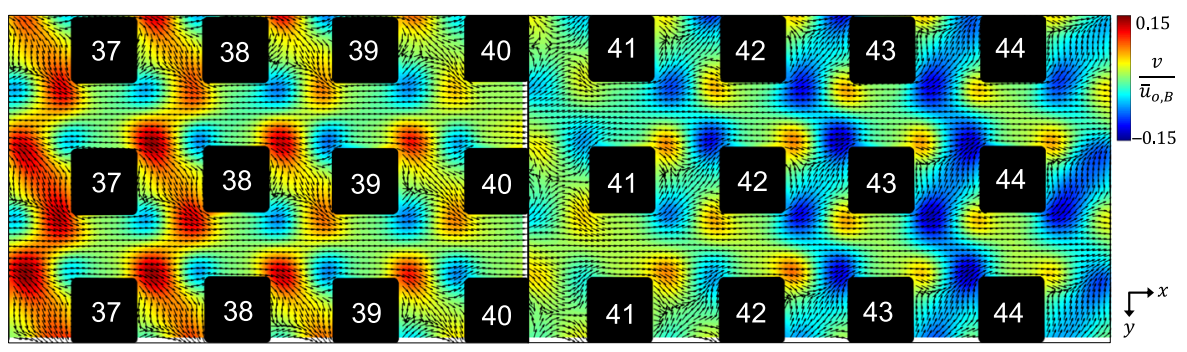

FIG. 8. Microscopic flow field (region D) in the middle of the micromodel (pillars 37-44) and far from the interface (pillar rows 16 th to 18 th) at $R e_{1}=0.14$. The $v$ velocity magnitude is normalised by the average freestream channel velocity in region $D, \bar{u}_{o, D}=0.3 \mathrm{~mm} / \mathrm{s}$. 


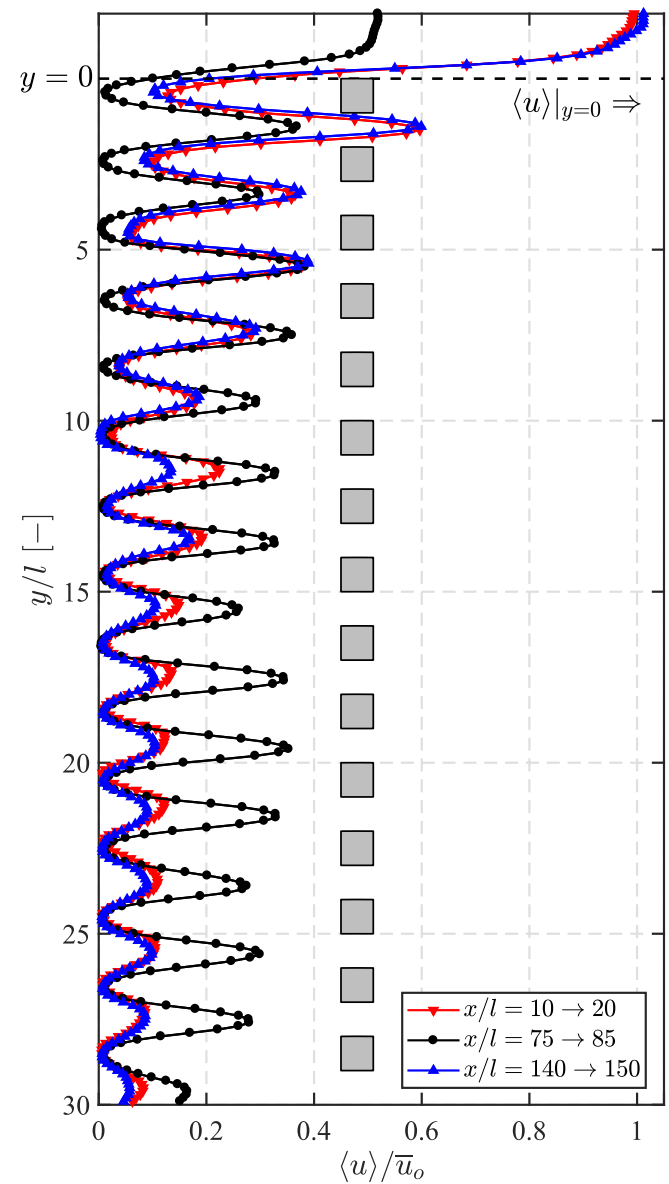

FIG. 9. Streamwise $u$-velocity profiles at three different axial positions indicate a slip velocity at the interface $(y=0)$.

that is similar to the one at the interface. However, before and after pillars 39 and 41, there is a clear tendency of downward and upward fluid motion, respectively, that was not observed in Fig. 7(b). This means that the length of the macroscopically parallel flow between the free-flow and the porous medium is reduced with increasing the distance from the interface. This is a direct effect of the triangular bottom of the micromodel that generates a $\mathrm{V}$-shaped flow distribution [see Fig. 4(b)].

\section{Interface boundary conditions}

\section{Velocity profiles}

Figure 9 shows the streamwise-averaged velocity profiles at various locations in the $x$-coordinate. These include the regions close to the inlet $(x / l=10-20)$, the outlet $(x / l=140-150)$, and the middle $(x / l=75-85)$ of the micromodel where the flow in the porous medium is macroscopically inward, outward, and parallel to the free-flow channel, respectively.

Due to the overall geometrical symmetry, the $\langle u\rangle$-velocity profiles close to the inlet and the outlet are nearly identical, showing gradually reduced velocity oscillations in the $y$-coordinate, with the maximum amplitude obtained between the pillars. In the free-flow regime, a laminar boundary layer is developed with a freestream velocity that is significantly higher than the oscillated velocities in the porous material. More specifically, the maximum $\langle u\rangle$ is gradually reduced from about $0.6 \bar{u}_{o}$ between the first two pillar rows to less than $0.1 \bar{u}_{o}$ at the bottom of the porous medium. These are the regions where the flow is characterised by a significant $v$-velocity component as the flow enters and escapes the porous medium, respectively (see Fig. 7). In the middle of the micromodel, on the other hand, the velocity oscillations are more pronounced, while the free-flow channel velocity is decelerated to compensate the continuity equation. As a result, the level of $\langle u\rangle$ in the porous medium is comparable to the channel freestream velocity, while a reduction of $\langle u\rangle$ at larger depths is not observed. Instead, the amplitude of $\langle u\rangle$ oscillations is about $65 \%$ of the freestream channel velocity in this region, and over the full depth of the porous material. This is the region where the flow is macroscopically parallel to the free-flow.

In all velocity profiles, however, a slip streamwise velocity, $\langle u\rangle_{y=0}$, can be observed at the interface between the free-flow and the porous medium. The slip velocity is relatively constant being about $20 \%-25 \%$ of the free-flow velocity at each individual streamwise position, that is, $0.1 / 0.5 \bar{u}_{o}(x=75-85)$ in the middle and $0.25 \bar{u}_{o}$ at the two edges of the micromodel.

\section{Interfacial coefficients}

The existence of a slip velocity at the porous medium and free-flow boundary demonstrates the possibility to derive the velocity slip and stress jump coefficients for the Beavers-Joseph ${ }^{18}$ and Ochoa-Tapia-Whitaker ${ }^{29}$ boundary conditions. The calculation of the interface coefficients $\alpha_{\mathrm{BJ}}$ and $\beta$ is based on a volumetric-averaged method, which has been extensively used in the literature in upscaling techniques. ${ }^{2,29,66-68}$ For the interface, the volume-averaging includes the first PIV interrogation window above and below the

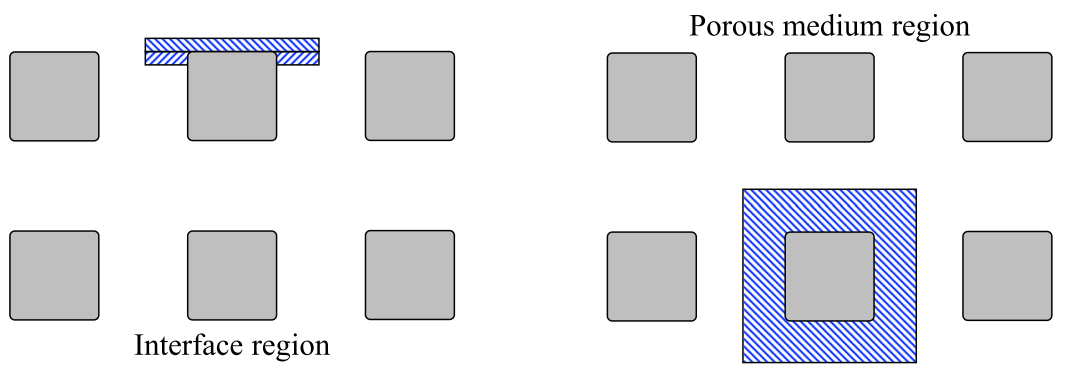

FIG. 10. Volume-averaging domains used for the calculation of velocity-slip and stress-jump interface coefficients. 
nominal interface which is assumed to be the top of the first pillar row, as shown in Fig. 10. For the Darcy velocity, on the other hand, the volume-averaging includes the flow region around each individual pillar at the bottom of the porous medium. Note also that the micro-PIV interrogation areas and window overlaps (see Sec. II B) were spatially selected so that any possible effects on the interfacial velocities are negligible. ${ }^{22}$ Therefore, the obtained data are adequately accurate to provide a fair estimation of the derived interface coefficients.

The velocity slip, $\alpha_{\mathrm{BJ}}$, and stress jump, $\beta$, constants are shown in Fig. 11. On the left side, the coefficients are plotted against the length of the porous medium, where the flow direction is gradually varied from $90^{\circ}$ to $-90^{\circ}$, as shown in Fig. 4(b). Therefore, similar to the streamwise velocity distributions in Fig. 5, the variations of $\alpha_{\mathrm{BJ}}$ and $\beta$ can be also classified into three different regions. These include the two regions where the spanwise $v$-velocity component is significant as the flow enters and escapes the porous structure, e.g., oblique flow at the interface, and the region of macroscopically parallel flow. Regarding the latter, Fig. 11(a) shows that $\alpha_{\mathrm{BJ}}$ remains

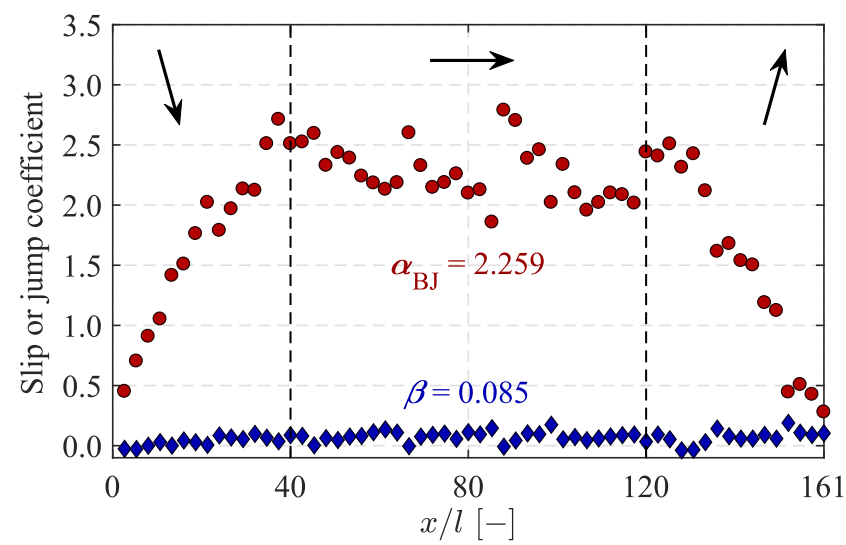

(a)

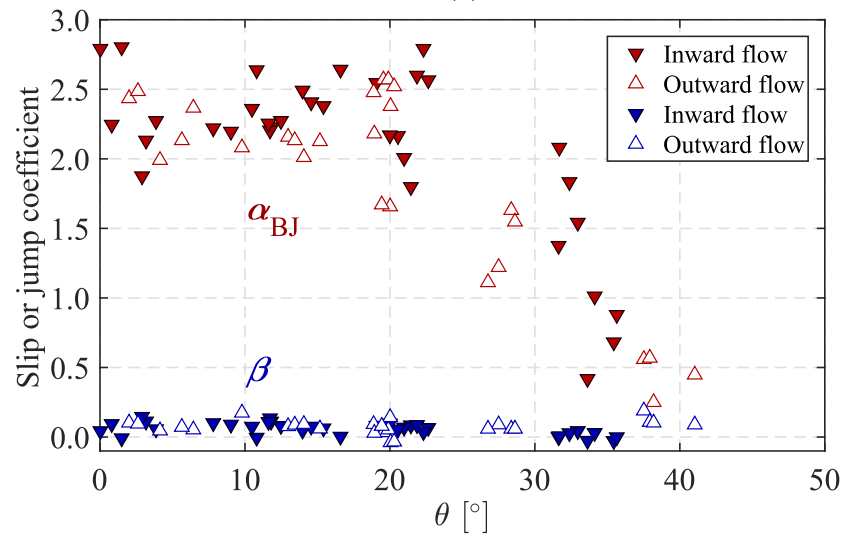

(b)

FIG. 11. Interface velocity slip and stress jump coefficients calculated based on the Beavers-Joseph-Saffman ${ }^{18,19}$ and Ochoa-Tapia-Whitaker boundary conditions ${ }^{28,29}$ as a function of (a) streamwise position and (b) flow direction at $R e_{l}=0.14$.

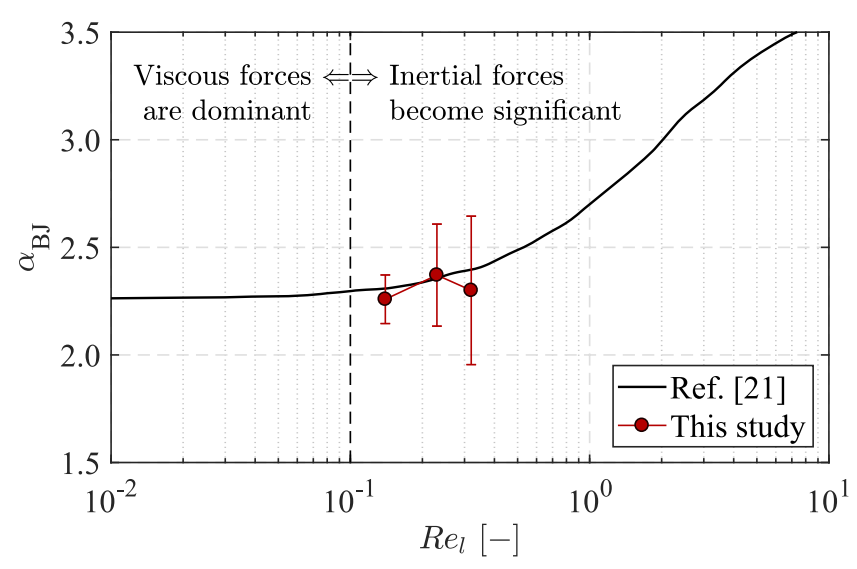

FIG. 12. Beavers and Joseph slip coefficient $\alpha_{B J}$ as a function of Reynolds number based on the pore length scale $I$ and channel average free-stream velocity $\bar{u}_{o}$.

essentially constant from $x / \alpha=40$ to 120 , reaching an average value of 2.259. However, at the regions where non-parallel flow conditions exist, $\alpha_{\mathrm{BJ}}$ significantly decreases to 0.5 . The coefficient $\beta$, on the other hand, is not affected by the streamwise position and remains essentially constant with an average value of 0.085 . This value is within the range reported in the literature, ${ }^{29}$ e.g., $-1<\beta<1$. Apparently, the flow direction at the interface influences dramatically the level of $\alpha_{\mathrm{BJ}}$, while $\beta$ remains unaffected. The obtained trends are better visualised in Fig. 11(b) which shows the interface coefficients as a function of flow angle for both "inward" and "outward," towards the porous medium, flow directions. At moderate flow angles, e.g., $\theta<30^{\circ}, \alpha_{\mathrm{BJ}}$ is not influenced by the oblique interface flow. However, the velocity slip coefficient significantly decreases as the flow substantially deviates from a typical Poiseuille flow $(\theta=0)$, which is in agreement with theoretical literature models. ${ }^{21}$ Interestingly, no differences are observed between "inward" and "outward" flow directions demonstrating a similar behavior as the flow enters or escapes the porous structure.

Regarding the influence of free-flow velocity, Fig. 12 shows the variation of $\alpha_{\mathrm{BJ}}$ with respect to the Reynolds number based on the pillar size $l$. The experiments took place in a Reynolds number regime slightly above 0.1 , where inertial effects start becoming significant, and hence, $\alpha_{\mathrm{BJ}}$ is slightly increased. ${ }^{21}$ The results show nearly no dependence of $\alpha_{\mathrm{BJ}}$ with the $R e_{l}$ in the range of $0.14-0.33$, and the differences lie within the experimental uncertainties. However, very good agreement with literature models is observed since the average value of $\alpha_{\mathrm{BJ}}$ in this flow regime is 2.3.

\section{CONCLUSIONS}

In this study, single-phase microfluidic experiments were carried out to reveal the full velocity field in a free-flow and porous media coupled system at creeping flow conditions $(R e<1)$. The results showed that the flow field is macroscopically characterised by a typical U-shaped Stokes distribution where the flow in the middle of the porous medium is parallel to the free-flow. However, a microscopic interpretation of the flow physics demonstrated that parallel flow at the interface does not exist, even if the 
advective inertial forces are considerably smaller than the viscous forces. Instead, the interface flow field is characterised by velocity oscillations where the free-flow periodically crosses the nominal interface $(y=0)$, generating small-scale U-shaped flow profiles between the pores of the first pillar row. Nevertheless, the volumeaveraging velocity profiles indicated a slip velocity at the interface over the full length of the porous medium. For a porous medium that consists of evenly spaced micro-structured rectangular pillars arranged in a uniform pattern (porosity 0.75 ), the Beavers-Joseph-Saffman velocity-slip coefficient is $\alpha_{\mathrm{BJ}}=2.3$, and the Ochoa-Tapia-Whitaker stress-jump coefficient is $\beta=0.085$. The latter showed no sensitivity with the interface flow angle, while $\alpha_{\mathrm{BJ}}$ decreases as the flow significantly deviates from a typical Poiseuille flow.

Finally, it should also be mentioned that the derived values of $\alpha_{\mathrm{BJ}}$ and $\beta$ are valid over a narrow range of low velocities, while the single-phase characteristics of the flow, and hence the absence of capillary effects, allow their extension at reasonably larger scales provided that the Reynolds numbers are maintained at the same level. On the other hand, different porous systems (e.g., staggered or randomly distributed pillars) will definitely influence the microscopic characteristics of the flow, and thus, other transport processes such as convective heat transfer. However, the theoretical treatment on the macro- or the REV-scale will result in similar outcomes for the momentum transfer, provided that the permeability and the roughness of the irregular porous medium are the same. Plausible extensions of the current work will therefore involve experiments to examine the validity of interface boundary conditions for turbulent multiphase flows.

\section{ACKNOWLEDGMENTS}

Alexandros Terzis would like to acknowledge the Alexander von Humboldt foundation for the funding support. This research was also funded by the German Research Foundation (DFG) in the framework of SFB-1313, Research Project No. A.02. The authors are also grateful to Dr. David Sebastián Martínez Hernández from University of Stuttgart for the fruitful discussions.

\section{REFERENCES}

${ }^{1}$ E. Shahraeeni, P. Lehmann, and D. Or, "Coupling of evaporative fluxes from drying porous surfaces with air boundary layer: Characteristics of evaporation from discrete pores," Water Resour. Res. 48(9), W09525, https://doi.org/10.1029/ 2012wr011857 (2012).

${ }^{2}$ V. P. Chauhan, T. Stylianopoulos, Y. Boucher, and R. K. Jain, "Delivery of molecular and nanoscale medicine to tumors: Transport barriers and strategies," Annu. Rev. Chem. Biomol. Eng. 2(1), 281-298 (2011).

${ }^{3}$ V. Gurau, H. Liu, and S. Kakaç, "Two-dimensional model for proton exchange membrane fuel cells," AIChE J. 44(11), 2410-2422 (1998).

${ }^{4}$ W. Dahmen, T. Gotzen, S. Müller, and M. Rom, "Numerical simulation of transpiration cooling through porous material," Int. J. Numer. Methods Fluids 76(6), 331-365 (2014).

${ }^{5}$ P. Verboven, D. Flick, B. M. Nicolaï, and G. Alvarez, "Modelling transport phenomena in refrigerated food bulks, packages and stacks: Basics and advances," Int. J. Refrig. 29(6), 985-997 (2006).

${ }^{6}$ M. Mößner and R. Radespiel, "Flow simulations over porous mediaComparisons with experiments," Comput. Fluids 154, 358-370 (2017).

${ }^{7} \mathrm{~J}$. Bear, Dynamics of Fluids in Porous Media (Dover Publishing, 1972), ISBN: 10: 0-486-65675-6.
${ }^{8}$ H. C. Brinkman, "A calculation of the viscous force exerted by a flowing fluid on a dense swarm of particles," Appl. Sci. Res. 1, 27 (1949).

${ }^{9}$ B. Goyeau, D. Lhuillier, D. Gobin, and M. G. Velarde, "Momentum transport at a fluid-porous interface," Int. J. Heat Mass Transfer 46(21), 4071-4081 (2003).

${ }^{10} \mathrm{R}$. Rosenzweig and U. Shavit, "The laminar flow field at the interface of a Sierpinski carpet configuration," Water Resour. Res. 43(10), 1395, https://doi.org/10.1029/2006wr005801 (2007).

${ }^{11}$ D. A. Nield, "The limitations of the Brinkman-Forchheimer equation in modeling flow in a saturated porous medium and at an interface," Int. J. Heat Fluid Flow 12(3), 269-272 (1991).

${ }^{12}$ N. Martys, D. P. Bentz, and E. J. Garboczi, "Computer simulation study of the effective viscosity in Brinkman's equation," Phys. Fluids 6(4), 1434-1439 (1998).

${ }^{13}$ L. Durlofsky and J. F. Brady, "Analysis of the Brinkman equation as a model for flow in porous media," Phys. Fluids 30(11), 3329 (1998).

${ }^{14} \mathrm{~A}$. J. Basu and A. Khalili, "Computation of flow through a fluid-sediment interface in a benthic chamber," Phys. Fluids 11(6), 1395-1405 (1999).

${ }^{15}$ K. Mosthaf, K. Baber, B. Flemisch, R. Helmig, A. Leijnse, I. Rybak, and B. Wohlmuth, "A coupling concept for two-phase compositional porous-medium and single-phase compositional free flow," Water Resour. Res. 47(10), W10522, https://doi.org/10.1029/2011wr010685 (2011).

${ }^{16}$ M. Hassanizadeh and W. G. Gray, "General conservation equations for multiphase systems: 1. Averaging procedure," Adv. Water Resour. 2, 131-144 (1979).

${ }^{17} \mathrm{Z}$. Wu and P. Mirbod, "Experimental analysis of the flow near the boundary of random porous media," Phys. Fluids 30(4), 047103 (2018).

${ }^{18}$ G. S. Beavers and D. D. Joseph, "Boundary conditions at a naturally permeable wall,” J. Fluid Mech. 30(1), 197-207 (1967).

${ }^{19}$ P. G. Saffman, "On the boundary condition at the surface of a porous medium," Stud. Appl. Math. 50(2), 93-101 (1971).

${ }^{20}$ R. E. Larson and J. J. L. Higdon, "Microscopic flow near the surface of twodimensional porous media. Part 2. Transverse flow," J. Fluid Mech. 178(-1), 119-136 (1987).

${ }^{21}$ M. Sahraoui and M. Kaviany, "Slip and no-slip velocity boundary conditions at interface of porous, plain media," Int. J. Heat Mass Transfer 35(4), 927-943 (1992).

${ }^{22}$ J. K. Arthur, D. W. Ruth, and M. F. Tachie, "Porous medium flow and an overlying parallel flow: PIV interrogation area and overlaps, interfacial location, and depth ratio effects," Transp. Porous Media 97(1), 5-23 (2013).

${ }^{23}$ G. S. Beavers, E. M. Sparrow, and R. A. Magnuson, "Experiments on coupled parallel flows in a channel and a bounding porous medium," J. Basic Eng. 92(4), 843-848 (1970).

${ }^{24}$ G. S. Beavers, E. M. Sparrow, and B. A. Masha, "Boundary condition at a porous surface which bounds a fluid flow," AIChE J. 20(3), 596-597 (1974).

${ }^{25}$ D. F. James and A. M. J. Davis, "Flow at the interface of a model fibrous porous medium," J. Fluid Mech. 426, 47-72 (2001).

${ }^{26}$ G. I. Taylor, "A model for the boundary condition of a porous material. Part 1," J. Fluid Mech. 49(2), 319-326 (1971).

${ }^{27}$ S. Richardson, "A model for the boundary condition of a porous material. Part 2," J. Fluid Mech. 49(2), 327-336 (1971).

${ }^{28} \mathrm{~J}$. A. Ochoa-Tapia and S. Whitaker, "Momentum transfer at the boundary between a porous medium and a homogeneous fluid-I. Theoretical development," Int. J. Heat Mass Transfer 38(14), 2635-2646 (1995).

${ }^{29} \mathrm{~J}$. A. Ochoa-Tapia and S. Whitaker, "Momentum transfer at the boundary between a porous medium and a homogeneous fluid-II. Comparison with experiment," Int. J. Heat Mass Transfer 38(14), 2647-2655 (1995).

${ }^{30} \mathrm{M}$. Chandesris and D. Jamet, "Boundary conditions at a planar fluid-porous interface for a Poiseuille flow," Int. J. Heat Mass Transfer 49(13-14), 2137-2150 (2006).

${ }^{31} \mathrm{M}$. Chandesris and D. Jamet, "Boundary conditions at a fluid-porous interface: An a priori estimation of the stress jump coefficients," Int. J. Heat Mass Transfer 50(17-18), 3422-3436 (2007).

${ }^{32}$ K. Baber, K. Mosthaf, B. Flemisch, R. Helmig, S. Müthing, and B. Wohlmuth, "Numerical scheme for coupling two-phase compositional porous-media flow and one-phase compositional free flow," IMA J. Appl. Math. 77(6), 887-909 (2012). 
${ }^{33}$ R. Helmig, B. Flemisch, M. Wolff, A. Ebigbo, and H. Class, "Model coupling for multiphase flow in porous media," Adv. Water Resour. 51, 52-66 (2013).

${ }^{34}$ V. A. Jambhekar, R. Helmig, N. Schröder, and N. Shokri, "Free-flow-porousmedia coupling for evaporation-driven transport and precipitation of salt in soil," Transp. Porous Media 110(2), 251-280 (2015).

${ }^{35} \mathrm{R}$. Masson, L. Trenty, and Y. Zhang, "Coupling compositional liquid gas Darcy and free gas flows at porous and free-flow domains interface," J. Comput. Phys. 321, 708-728 (2016).

${ }^{36}$ H. K. Moffatt, "Viscous and resistive eddies near a sharp corner," J. Fluid Mech. 18(1), 1-18 (1964).

${ }^{37} \mathrm{~J}$.- T. Jeong, "Slip boundary condition on an idealized porous wall," Phys. Fluids 13(7), 1884-1890 (2001).

${ }^{38} \mathrm{C}$. J. Heaton, "On the appearance of Moffatt eddies in viscous cavity flow as the aspect ratio varies," Phys. Fluids 20(10), 103102 (2008).

${ }^{39} \mathrm{X}$. Chu, B. Weigand, and V. Vaikuntanathan, "Flow turbulence topology in regular porous media: From macroscopic to microscopic scale with direct numerical simulation," Phys. Fluids 30(6), 065102 (2018).

${ }^{40} \mathrm{X}$. Chu, G. Yang, S. Pandey, and B. Weigand, "Direct numerical simulation of convective heat transfer in porous media," Int. J. Heat Mass Transfer 133, 11-20 (2019).

${ }^{41}$ G. Yang, B. Weigand, A. Terzis, K. Weishaupt, and R. Helmig, "Numerical simulation of turbulent flow and heat transfer in a three-dimensional channel coupled with flow through porous structures," Transp. Porous Media 122(1), 145-167 (2018).

${ }^{42}$ S. K. Gupte and S. G. Advani, "Flow near the permeable boundary of a porous medium: An experimental investigation using LDA," Exp. Fluids 22(5), 408-422 (1997).

${ }^{43}$ M. F. Tachie, D. F. James, and I. G. Currie, "Slow flow through a brush," Phys. Fluids 16(2), 445-451 (2004).

${ }^{44}$ P. Prinos, D. Sofialidis, and E. Keramaris, "Turbulent flow over and within a porous bed," J. Hydraul. Eng. 129(9), 720-733 (2003).

${ }^{45}$ M. F. Tachie, D. F. James, and I. G. Currie, "Velocity measurements of a shear flow penetrating a porous medium," J. Fluid Mech. 493, 319-343 (2003).

${ }^{46}$ A. Goharzadeh, A. Khalili, and B. B. Jørgensen, "Transition layer thickness at a fluid-porous interface," Phys. Fluids 17(5), 057102 (2005).

${ }^{47}$ J. K. Arthur, D. W. Ruth, and M. F. Tachie, "PIV measurements of flow through a model porous medium with varying boundary conditions," J. Fluid Mech. 629, 343 (2009).

${ }^{48}$ J. M. K. Ng, I. Gitlin, A. D. Stroock, and G. M. Whitesides, "Components for integrated poly(dimethylsiloxane) microfluidic systems," Electrophoresis 23(20), 3461-3473 (2002).

${ }^{49} \mathrm{~J}$. Friend and L. Yeo, "Fabrication of microfluidic devices using polydimethylsiloxane," Biomicrofluidics 4(2), 026502 (2010).

${ }^{50}$ N. K. Karadimitriou, M. Musterd, P. J. Kleingeld, M. T. Kreutzer, S. M. Hassanizadeh, and V. J. Niasar, "On the fabrication of PDMS micromodels by rapid prototyping, and their use in two-phase flow studies," Water Resour. Res. 49(4), 2056-2067, https://doi.org/10.1002/wrcr.20196 (2013).
${ }^{51}$ I. M. Zarikos, S. M. Hassanizadeh, L. M. van Oosterhout, and W. van Oordt, "Manufacturing a micro-model with integrated fibre optic pressure sensors," Transp. Porous Media 122(1), 221-234 (2018).

${ }^{52}$ T. Jiang, Y. Ren, W. Liu, D. Tang, Y. Tao, R. Xue, and H. Jiang, "Dielectrophoretic separation with a floating-electrode array embedded in microfabricated fluidic networks," Phys. Fluids 30(11), 112003 (2018).

${ }^{53}$ Y. Ren, W. Liu, Z. Wang, and Y. Tao, "Induced-charge electrokinetics in rotating electric fields: A linear asymptotic analysis," Phys. Fluids 30(6), 062006 (2018).

${ }^{54}$ W. Liu, Y. Ren, Y. Tao, X. Chen, B. Yao, M. Hui, and L. Bai, "Control of two-phase flow in microfluidics using out-of-phase electroconvective streaming," Phys. Fluids 29(11), 112002 (2017).

${ }^{55}$ N. K. Karadimitriou, V. Joekar-Niasar, and O. G. Brizuela, "Hydro-dynamic solute transport under two-phase flow conditions," Sci. Rep. 7(1), 6624 (2017).

${ }^{56}$ B. Ling, M. Oostrom, A. M. Tartakovsky, and I. Battiato, "Hydrodynamic dispersion in thin channels with micro-structured porous walls," Phys. Fluids 30(7), 076601 (2018).

${ }^{57}$ J. G. Santiago, S. T. Wereley, C. D. Meinhart, D. J. Beebe, and R. J. Adrian, "A particle image velocimetry system for microfluidics," Exp. Fluids 25(4), 316-319 (1998).

${ }^{58}$ S. T. Wereley and C. D. Meinhart, "Recent advances in micro-particle image velocimetry,” Annu. Rev. Fluid Mech. 42(1), 557-576 (2010).

${ }^{59} \mathrm{O}$. Uzol and C. Camci, "The effect of sample size, turbulence intensity and the velocity field on the experimental accuracy of ensemble averaged PIV measurements," in 4th International Symposium on Particle Image Velocimetry, Gottingen, Germany, 17-19 September 2001.

${ }^{60}$ D. Michaelis, D. R. Neal, and B. Wieneke, "Peak-locking reduction for particle image velocimetry," Meas. Sci. Technol. 27(10), 104005 (2016).

${ }^{61}$ W. Thielicke and E. Stamhuis, "PIVlab-Towards user-friendly, affordable and accurate digital particle image velocimetry in MATLAB," J. Open Res. Software 2(1), 1202 (2014).

${ }^{62} \mathrm{~B}$. Wieneke, "PIV uncertainty quantification from correlation statistics," Meas. Sci. Technol. 26(7), 074002 (2015).

${ }^{63}$ I. Zarikos, A. Terzis, S. M. Hassanizadeh, and B. Weigand, "Velocity distributions in trapped and mobilized non-wetting phase ganglia in porous media," Sci. Rep. 8(1), 13228 (2018).

${ }^{64}$ C. Shen and J. M. Floryan, "Low Reynolds number flow over cavities," Phys. Fluids 28(11), 3191 (1985).

${ }^{65}$ P. N. Shankar and M. D. Deshpande, "Fluid mechanics in the driven cavity," Annu. Rev. Fluid Mech. 32, 93-136 (2000).

${ }^{66}$ F. J. Valdés-Parada, B. Goyeau, and J. Alberto Ochoa-Tapia, "Diffusive mass transfer between a microporous medium and an homogeneous fluid: Jump boundary conditions," Chem. Eng. Sci. 61(5), 1692-1704 (2006).

${ }^{67}$ W. P. Breugem, B. J. Boersma, and R. E. Uittenbogaard, "The laminar boundary layer over a permeable wall," Transp. Porous Media 59(3), 267-300 (2005).

${ }^{68}$ G. Yang, E. Coltman, K. Weishaupt, A. Terzis, R. Helmig, and B. Weigand, "On the Beavers-Joseph interface condition for non-parallel coupled channel flow over a porous structure at high Reynolds numbers," Transp. Porous Media (to be published). 